\title{
Fuel Consumption and Traffic Emissions Evaluation of Mixed Traffic Flow with Connected Automated Vehicles at Multiple Traffic Scenarios
}

\author{
Bin Zhao, ${ }^{1,2,4}$ Yalan Lin, ${ }^{1,2}$ Huijun Hao, ${ }^{1,2}$ and Zhihong Yao $\mathbb{D}^{1,2,3,4}$ \\ ${ }^{1}$ School of Transportation and Logistics, Southwest Jiaotong University, Chengdu, Sichuan 610031, China \\ ${ }^{2}$ National Engineering Laboratory of Integrated Transportation Big Data Application Technology, Southwest Jiaotong University, \\ Chengdu, Sichuan 611756, China \\ ${ }^{3}$ Institute of System Science and Engineering, Southwest Jiaotong University, Chengdu, Sichuan 611756, China \\ ${ }^{4}$ National United Engineering Laboratory of Integrated and Intelligent Transportation, Southwest Jiaotong University, Chengdu, \\ Sichuan 611756, China
}

Correspondence should be addressed to Zhihong Yao; zhyao@swjtu.edu.cn

Received 6 October 2021; Accepted 29 December 2021; Published 12 January 2022

Academic Editor: Wei Zhang

Copyright (c) 2022 Bin Zhao et al. This is an open access article distributed under the Creative Commons Attribution License, which permits unrestricted use, distribution, and reproduction in any medium, provided the original work is properly cited.

To analyze the impact of different proportions of connected automated vehicles (CAVs) on fuel consumption and traffic emissions, this paper studies fuel consumption and traffic emissions of mixed traffic flow with CAVs at different traffic scenarios. Firstly, the car-following modes and proportional relationship of vehicles in the mixed traffic flow are analyzed. On this basis, different car-following models are applied to capture the corresponding car-following modes. Then, Virginia Tech microscopic (VT-micro) model is adopted to calculate the instantaneous fuel consumption and traffic emissions. Finally, based on three typical traffic scenarios, a basic segment with bottleneck zone, ramp of the freeway, and signalized intersection, a simulation platform is built based on Python and SUMO to obtain vehicle trajectory data, and the fuel consumption and traffic emissions in different scenarios are obtained. The results show that (1) In different traffic scenarios, the application of CAVs can reduce fuel consumption and traffic emissions. The higher the penetration rate, the more significant the reduction in fuel consumption and traffic emissions. (2) In the three typical traffic scenarios, the advantages of CAVs are more evident in the signalized intersection. When the penetration rate of CAVs is $100 \%$, the fuel consumption and traffic emissions reduction ratio is as high as $32 \%$. It is noteworthy that the application of CAVs in urban transportation will significantly reduce fuel consumption and traffic emissions.

\section{Introduction}

With the development of the urban economy, the number of vehicles has increased rapidly. Traffic congestion has become more and more serious $[1,2]$, and also caused massive fuel consumption and traffic emissions [3]. In 2018, the United States consumed approximately 143 billion gallons of motor gasoline, with a daily average of 391 million gallons [4]. Thence, it is a top priority to reduce fuel consumption and emissions of traffic systems.

Autonomous driving technology is a comprehensive application of traffic monitoring, route navigation, and artificial intelligence technology. The development of connected automated transportation systems has shown a trend of integrating with autonomous driving technology. Therefore, CAVs $[5,6]$ will cause the transformation of intelligent transportation systems [7]. In the connected automated transportation system, the preceding vehicle can transmit position, speed, acceleration, and other information to the following vehicle in real-time through Vehicle-toVehicle (V2V) communication technology. At this time, CAVs can drive in cooperative adaptive cruise control (CACC) mode through V2V communication technology [8]. In addition, if $\mathrm{V} 2 \mathrm{~V}$ communication conditions are not met, CAVs will drive in adaptive cruise control (ACC) mode [9]. To sum up, the widespread application of CAVs will 
effectively reduce the response delay of vehicles to changes in surrounding traffic conditions and shorten the car-following time between vehicles. Therefore, CAVs can improve the inherent characteristics of traffic flow, and achieve a comprehensive breakthrough in alleviating traffic system congestion, energy-saving, and traffic emission reduction. Many scholars generally believe that the CAVs are expected to improve the quality of traffic flow from the microscopic traffic flow level $[10,11]$, and provide an effective way to bring down fuel consumption and traffic emissions.

In recent years, lots of studies have been focused on fuel consumption and emissions of the traffic system [12]. How to calculate the fuel consumption and emission of vehicles have always been a significant research topic. Therefore, some research focused on fuel consumption and emission models.

As the earliest generation of fuel consumption and emission factor model of motor vehicles, the Emission Factors (EMFAC) model [13] is developed by the California Air Resources Bureau. In addition, the European Environment Agency also funded the development of the computer program to calculate emissions from the road transport (COPERT) model [14]. Compared with EMFAC, the COPERT model has lower requirements on parameters, including 15 parameters such as fleet composition, average driving speed, average journey length, and fuel parameters, but its coverage is not as wide as EMFAC. Due to the design requirements for emissions, micro fuel consumption and emission models appeared. The Parametric Analytical Model of Vehicle Energy Consumption (PAMVEC) [15] calculates vehicle energy consumption based on total vehicle mass, parameter driving cycle description, and other attributes of the vehicle platform. The most intuitive description of the driving state of a motor vehicle is to establish a speed-acceleration matrix. The value of the matrix is the average fuel consumption level corresponding to the speed and acceleration. On this basis, Cernuschi et al. [16] divided the data into five types: high deceleration, low deceleration, uniform speed, high acceleration, and low acceleration. Meanwhile, the regression analysis method is used to fit best the emission speed curve of each type of acceleration mode, and the idle fuel consumption and emission are analyzed. Subsequently, under the premise of combining speed and acceleration with different power products, Ahn [17] proposed the VT-micro model, which determines the value of fuel consumption and different emission indicators based on the combination of different power products of speed and acceleration. With the development of CAVs, many researchers have begun to study the impact of CAVs on fuel consumption and traffic emissions. On this basis, Zegeye et al. provided a general framework to integrate macroscopic traffic flow models with microscopic fuel consumption and emission models, and extended the VT-micro model to the macroscopic traffic flow model to further shorten the simulation time and carry out fairly accurate estimates of the emissions and fuel consumption. Chandra and Camal [18] used simulation methods to calculate the fuel consumption and emissions of connected vehicle technology (CVT). The results indicated that the CVT reduces fuel consumption and emissions in different traffic conditions. Aiming at signalized intersections, Han et al. [19] used the trajectory optimization method, PTO-GFC, to study the fuel consumption and emissions of CAVs passing intersections under smooth trajectories. Research results showed that fuel consumption and emissions will be reduced to varying degrees under the proposed method. Yao et al. [6] studied the impact of mixed traffic flow at a single intersection on fuel consumption and emissions, and proposed a joint optimization framework for traffic signals and vehicle trajectories at a single intersection, including HDVs and CAVs. The results showed that this method could reduce fuel consumption and emissions. Yao and Li [20] applied a more detailed micro-fuel model (e.g., VT-micro model) in the study of CAVs trajectory optimization at an isolated signalized intersection with a single-lane road. Then, a joint objective of travel time, fuel consumption, and safety is implemented to improve traffic mobility, energy efficiency, and safety simultaneously. Aiming at the expressway ramp, Qin et al. [21] proposed a stability analysis method applied to mixed traffic flow, which simulates the mixed traffic flow of the expressway ramp segment to evaluate the impact of stability on fuel consumption and traffic emissions. Subsequently, the correlation between CACC vehicles improving traffic flow stability and reducing traffic emissions and fuel consumption was further studied. A simulation was applied to evaluate the changes in fuel consumption and emissions under traffic oscillation. The results showed that stability could qualitatively affect the reduction of fuel consumption and emissions, and provide new ideas for reducing fuel consumption and traffic emissions [22].

The above analysis indicated that the current research on fuel consumption and emission models is relatively mature. With the development of CAVs, related scholars have made useful explorations on the fuel consumption and emissions of the transportation system. However, to our best knowledge, there are some issues that need to be further discussed: (1) Some studies only focus on fuel consumption or emissions, and did not discuss the relationship between fuel consumption and traffic emissions under the same scenario. (2) Most researches on traffic scenarios are relatively single, and the impact mechanism of CAVs on fuel consumption and traffic emission under different traffic scenarios is not deeply discussed. (3) Most studies only focus on single-lane freeways. The scenarios do not involve lane-changing behavior, and the impact of vehicle lane-changing on fuel consumption and emission is not considered. Therefore, the impact of CAVs on the fuel consumption and traffic emissions of mixed traffic flows in different traffic scenarios remains to be revealed.

To address these issues, this paper is dedicated to evaluating the impact of the penetration rate of CAVs on the fuel consumption and traffic emission of mixed traffic flow under three classic traffic scenarios: basic road segment with bottleneck zone, a ramp of the freeway, and a signalized intersection. Firstly, the microscopic driving behavior and three car-following modes of mixed traffic flow are analyzed. Secondly, three car-following models were applied to describe these three car-following modes, 
and the lane-changing model LC2013 [23] was adopted for different traffic scenarios. Then, the VT-micro fuel consumption and emission model is introduced to calculate fuel consumption and traffic emissions in different traffic scenarios. Finally, based on a simulation experiment with SUMO, three traffic scenarios are simulated, and trajectory data are obtained. Based on the VT-micro model, fuel consumption and traffic emissions in different scenarios are calculated and analyzed. Thence, the main contributions of this work are as follows.

(1) The car-following behavior of vehicles in the mixed traffic flow is discussed, and different car-following models are used to describe the car-following characteristics in the mixed traffic flow.

(2) The impact of lane changing on fuel consumption and emission is considered, and lane-changing model LC2013 [23] is applied to different traffic scenarios.

(3) The influence of the penetration rate of CAVs on the fuel consumption and emission of mixed traffic flow under different traffic scenarios is studied.

The remainder of this paper is as follows. The micro-driving behavior, the car-following model, the car-following model, and the lane-changing model are analyzed in Section 2. Section 3 describes fuel consumption and emission models. Simulation analysis was conducted in Section 4, including simulation environment construction and simulation results analysis under different traffic scenarios. Finally, Section 5 summarizes the whole paper and proposes future work.

\section{Micro Driving Behavior Analysis}

\subsection{Mixed Traffic Flow}

2.1.1. Car-Following Modes. Considering that it will take a long time to upgrade and deploy CAVs, there will be a mixture of CAVs and HDVs on the road in the future $[5,24,25]$. As shown in Figure 1, there are four car-following modes: (i) HDV-HDV, (ii) HDV-CAV, (iii) CAV-HDV, and (iv) CAV-CAV.

(1) Modes 1 and 2. As shown in Figure 1, the current vehicle of modes 1 and 2 is an HDV. When the front vehicle changes driving behavior (e.g., acceleration and deceleration), the driver of the current vehicle needs to perceive, identify, judge, and take action. Thence, car-following behavior is determined by the human driver.

(2) Mode 3. In this mode, the current and front vehicles are CAV and HDV, respectively. The current vehicle is equipped with the onboard sensing systems (e.g., laser, radar, camera), which can quickly and accurately capture the front vehicle's driving behavior. This mode is called adaptive cruise control (ACC). The ACC system makes the current vehicle closely follow the front vehicle via controlling the accelerator and brake of the vehicle based on the advanced driving assistance system (ADAS).

(3) Mode 4. In this mode, the current and front vehicles are CAVs. The current vehicle can interact with the front vehicle via V2V communication. Therefore, the current vehicle can make synchronous driving behavior changes with the front vehicle. This mode is called cooperative adaptive cruise control (CACC). CACC system can shorten the distance between vehicles as much as possible on the premise of ensuring safety, to improve the traffic capacity of the transportation system.

2.1.2. Proportional Analysis. If it is assumed that the penetration rate of CAVs in the mixed traffic flow is $p$, then the proportion of HDVs is $1-p$. Thus, the proportions of carfollowing modes 1 and 2 are $1-p$. According to probability theory, the proportions of car-following modes 3 and 4 are $p(1-p)$ and $p^{2}$, respectively. To sum up, the proportions of different car-following modes are shown in Table 1.

2.2. Car-Following Model. In this paper, the intelligent driver model (IDM) [26], ACC, and CACC models [27, 28] are applied to describe the car-following behaviors of HDVs and CAVs. As shown in Figure 1, the IDM is utilized to simulate modes 1 and 2, and the ACC and CACC are applied to capture modes 3 and 4, respectively. The detailed description of the three car-following models is as follows.

2.2.1. Intelligent Driver Model. In the existing research, the models describing the driver's car-following behavior mainly include stimulus-response model, safety distance model, social force model, and optimal speed model. As a kind of social force model, the IDM [26] can accurately describe the car-following behavior of drivers, and is widely used in the study of micro traffic flow. Therefore, the IDM model is adopted in this paper to describe the car-following behavior of HDVs. The model is defined by equation.

$$
a_{i, k+1}=\bar{a}_{i}\left[1-\left(\frac{v_{i, k}}{v_{f}}\right)^{\delta}-\left(\frac{s^{*}\left(v_{i, k}, \Delta v_{i, k}\right)}{s_{i}}\right)^{2}\right] \text {, }
$$

where $a_{i, k+1}$ is the acceleration of the $i$-th vehicle at $k+1$ time step; $\bar{a}_{i}$ is the desired acceleration of the $i$-th vehicle; $v_{f}$ is the free flow velocity; $s^{*}$ is the expected distance between the $i$ - 1-th and -th vehicles; $v_{i, k}$ is the speed of the $i$-th vehicle at $k$ time step; $\Delta v_{i, k}$ is the speed difference between the $i-1$-th and $i$-th vehicles at the $k$ time step; $\Delta v_{i, k}=v_{i, k}-v_{i-1, k} ; s_{i}$ is the actual distance between the $i-1$-th and $i$-th vehicles.

The expected distance $s^{*}$ is determined by the speed of the $i$-th vehicle and the speed difference of the preceding car, and the calculation equation is:

$$
s^{*}\left(v_{i, k}, \Delta v_{i, k}\right)=s_{0}+T v_{i, k}+\frac{v_{i, k} \Delta v_{i, k}}{2 \sqrt{\bar{a}_{i} \bar{b}_{i}}},
$$

where $s_{0}$ is the minimum headway when stationary; $T$ is the safe time headway; $\bar{b}_{i}$ is the comfortable deceleration of the $i$-th vehicle. Referring to Treiber et al. [26], the parameters of IDM are: $\bar{a}_{i}=1 \mathrm{~m} / \mathrm{s}^{2}, \bar{b}_{i}=2 \mathrm{~m} / \mathrm{s}^{2}, T=1.5 \mathrm{~s}, v_{f}=33.3 \mathrm{~m} / \mathrm{s}$, and $s_{0}=2 \mathrm{~m}$. 


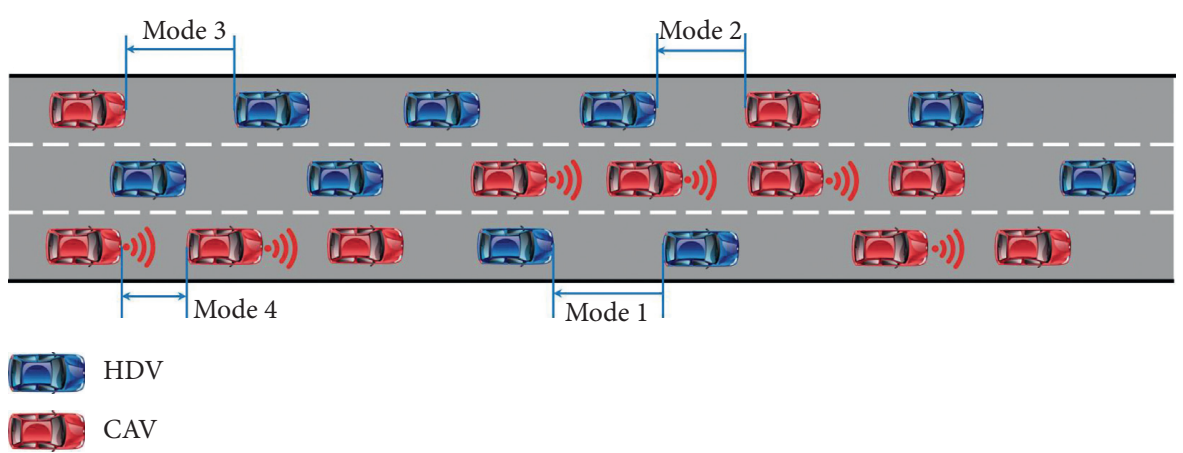

FIgURE 1: Four car-following modes of the mixed traffic flow.

Table 1: The ratio of the four modes.

\begin{tabular}{lccc}
\hline \multicolumn{2}{l}{ Modes } & Car-following models & Proportion \\
\hline 1 & HDV-HDV & HDV & $1-p$ \\
2 & HDV-CAV & ACC & $p(1-p)$ \\
3 & CAV-HDV & CACC & $p^{2}$ \\
4 & CAV-CAV &
\end{tabular}

2.2.2. Adaptive Cruise Control Model. The ACC system makes the current vehicle closely follow the front vehicle via controlling the accelerator and brake of the vehicle based on the advanced driving assistance system (ADAS). Generally, the ACC system will be equipped with sensing devices, such as laser, radar, camera, etc. These can measure the speed and position difference between the current vehicle and the front vehicle. The ACC system adjusts the current vehicle's speed to keep the desired headway between it and the front vehicle. In particular, when there is no front vehicle, the ACC system will cruise at the designed speed.

The ACC car-following model $[27,28]$ developed and validated with field data is adopted in this paper. The integrated ACC car-following model is divided into four modes: (i) speed control, (ii) gap control, (iii) gap-closing control, and (iv) collision avoidance. The fourth mode (i.e., collision avoidance) is developed by TransAID [23], which tries to avoid rear-end collisions between adjacent vehicles. The details of the four control modes are discussed below.

(1) Speed Control. The speed control mode aims to keep the preset speed of the vehicle. When the vehicle has no preceding vehicle within the coverage of the sensor or the distance from the preceding vehicle is more significant than $120 \mathrm{~m}$, the speed control mode is activated [28]. The speed control mode calculates the acceleration of the $i$-th vehicle at $k$ time step and is given by the following equation.

$$
a_{i, k+1}=\beta_{1}\left(v_{d}-v_{i, k}\right) \text {, }
$$

where $v_{d}$ is the ideal velocity; $v_{i, k}$ is the velocity of the $i$ th vehicle at $k$ time step; and $\beta_{1}$ is the control gain, which determines the speed deviation rate of acceleration. Xiao et al. [28] suggested that the value is $0.3-0.4 s^{-1}$. In this paper, the value of $0.4 s^{-1}$ was selected.

(2) Gap Control. The gap control mode keeps a constant time gap between the vehicle equipped with ACC and the vehicle ahead. This mode is activated when the gap and speed deviations (vehicle in front) are, respectively, less than $0.2 \mathrm{~m}$ and $0.1 \mathrm{~m} / \mathrm{s}$. The acceleration of the $i$-th vehicle at time step $k+1$ is described by the second-order function based on the velocity and gap error with the leading vehicle, which is defined in the following equation.

$$
a_{i, k+1}=\beta_{2} e_{i, k}+\beta_{3}\left(v_{i-1, k}-v_{i, k}\right),
$$

where $e_{i, k}$ is the gap error of the $i$ th vehicle at $k$ time step; $\beta_{2}$ and $\beta_{3}$ are the control gain for position and velocity deviations. Referring to Xiao et al. [28], the calibration values of control parameters in equation (4) are $\beta_{2}=0.23 \mathrm{~s}^{-1}$ and $\beta_{3}=0.07 \mathrm{~s}^{-1}$. Furthermore, the gap error $e_{i, k}$ is given by

$$
e_{i, k}=x_{i-1, k}-x_{i, k}-\tau_{a} v_{i, k}
$$

where $x_{i-1, k}$ and $x_{i, k}$ are the positions of the $i-1$-th and the $i$-th vehicles at time step $k$, and the $\tau_{d}$ is the desired time gap for the ACC system; its value range is generally 1.1 to $2.2 \mathrm{~s}$. In this study, the value of $\tau_{a}$ is set to $1.3 \mathrm{~s}$.

(3) Closed Gap Control. The gap closure controller realizes the stable transition from speed control mode to gap control mode. This mode is activated when the distance to the vehicle in front is less than $100 \mathrm{~m}$. According to Xiao et al. [28], the gap closure control mode can be presented by setting parameters of equation (4) as $\beta_{2}=0.04 s^{-1}$ and $\beta_{3}=0.8 s^{-1}$. Specially, if the distance is between $100 \mathrm{~m}$ and $120 \mathrm{~m}$, ACC-equipped vehicles will retain the fore control mode to supply hysteresis in the control loop and stable transition between the two modes.

(4) Collision Avoidance Control. The collision avoidance controller is developed by the TransAID team to prevent vehicle rear-end collision under safe conditions. When the distance to the preceding vehicle is less than $100 \mathrm{~m}$, and the position gap deviation is negative, this mode is activated. Similar to gapclosing control, the collision avoidance control mode can be presented by setting parameters of equation (4) as $\beta_{2}=0.8 \mathrm{~s}^{-1}$ and $\beta_{3}=0.23 \mathrm{~s}^{-1}$. In simulation, these parameters can ensure the safe operation of ACC vehicles, even in critical events [23].

2.2.3. Cooperative Adaptive Cruise Model. Compared with the ACC system, the CACC system can communicate with each other through $\mathrm{V} 2 \mathrm{~V}$ communication. Therefore, the CACC system can further improve traffic safety and traffic 
efficiency. The integrated CACC car-following model is verified by the actual data based on the work of Milanés et al. [29] and Xiao et al. [28, 30]. Similar to the ACC system, the developed control algorithm in the CACC system is divided into four modes: (i) speed control, (ii) gap control, (iii) closed gap control, and (iv) collision avoidance control.

(1) Speed Control. The speed control mode aims to keep the desired speed predefined by the driver. This mode is activated when there are no preceding vehicles in the sensor coverage area or the time gap is greater than 2 seconds. The acceleration of the $i$-th vehicle at time step $k+1$ can be obtained by equation.

$$
a_{i, k+1}=\beta_{4}\left(v_{d}-v_{i, k}\right),
$$

where $\beta_{4}$ is the speed control gain. Referring to Milanés and Shladover [27], the value of $\beta_{4}$ is set as $0.4 \mathrm{~s}^{-1}$.

(2) Gap Control. The gap control mode keeps a constant time gap between the vehicle equipped with CACC and the vehicle ahead. This mode is activated when the gap and velocity deviations (vehicle in front) are, respectively, less than $0.2 \mathrm{~m}$ and $0.1 \mathrm{~m} / \mathrm{s}$. The gap control of the CACC model is based on the first-order transfer function of velocity and gap error, which can be defined in the following equation.

$$
v_{i, k}=v_{i, k}+\beta_{5} e_{i, k}+\beta_{6} \dot{e}_{i, k},
$$

where $\dot{e}_{i, k}$ is the first derivative of the gap error $e_{i, k}$. The values of $\beta_{5}$ and $\beta_{6}$ are set to $\beta_{5}=0.45 \mathrm{~s}^{-1}$ and $\beta_{6}=0.0125 \mathrm{~s}^{-1}$, respectively [31]. The first derivative of the gap error is given by

$$
\dot{e}_{i, k}=v_{i-1, k}-v_{i, k}-\tau_{d} a_{i, k}
$$

where $a_{i, t}$ is the acceleration at time $t$, and $\tau_{d}$ is the required time gap defined by the CACC controller. The value is 0.6 to $2.2 \mathrm{~s}$, and the value of $\tau_{d}$ in this study is set to $1.0 \mathrm{~s}$.

(3) Closed Gap Control. The gap closure control mode realizes a stable transition from the speed control mode to the gap control mode. This mode is activated when the time distance to the front of the vehicle is less than $1.5 \mathrm{~s}$. The control formula is consistent with equation (7), but the value of the gain coefficient parameter is inconsistent. Referring to Reference [23], the gain coefficient parameter values are $\beta_{5}=0.005 \mathrm{~s}^{-1}$ and $\beta_{6}=0.05 \mathrm{~s}^{-1}$.

(4) Collision Avoidance Control. The collision avoidance mode prevents vehicle rear-end collision under safe conditions. When the time gap is less than $1.5 \mathrm{~s}$, and the position gap deviation is negative, this mode is activated. The controller's logic is consistent with the gap control and could be expressed by changing the gain coefficients, which are $\beta_{5}=0.45 \mathrm{~s}^{-1}$ and $\beta_{6}=0.05 \mathrm{~s}^{-1}$ [23].

In summary, both ACC and CACC car-following models contain four control modes, and the parameter values of different control modes can be summarized as shown in Figure 2.

2.3. Lane-Change Model. The lane-change model built-in sumo, namely LC2013 [23], is adopted in this study. The LC2013 model shows three main reasons (strategy, cooperation, and tactics) for changing lanes (right or left) in each simulation time step. The self-vehicle initially checks whether the right lane change is mandatory or required according to the logic described in Figure 3. If the right lane change is not mandatory or unnecessary, the motivation for the left lane change is determined according to the same rules. For details of LC2013, refer to Mintsis [23].

\section{Fuel Consumption and Traffic Emissions Model}

A variety of fuel consumption and emissions models of traffic systems began to develop a hundred years ago $[17,32,33]$; J. N. [34]. Among them, the VT-Micro model $[17,32,35]$ calculates fuel consumption and emissions based on the instantaneous speed and acceleration of the vehicle. In addition, due to the simple structure of the VT-micro model, it is widely used to evaluate vehicle fuel consumption and emissions in traffic research $[21,36]$. Thence, this paper selects the VT-micro model to calculate the fuel consumption and emissions in the traffic system. The model can be described as follows:

$$
\ln \left(\mathrm{MOE}_{e}\right)=\sum_{i=0}^{3} \sum_{j=0}^{3} K_{i, j}^{e} v^{i} a^{j},
$$

where $M O E_{e}$ is the fuel consumption and emission rate of the vehicle. $i$ and $j$ are the exponential coefficients of speed and acceleration, respectively. $K_{i, j}^{e}$ is the regression coefficient under the power $i$ of speed and the power $j$ of acceleration. $v$ and $a$ are the vehicle's speed and acceleration, respectively.

Equation (9) was developed by Ahn et al. [32] to calculate fuel consumption (FC), hydrocarbon (HC), carbon monoxide (CO), and oxides of nitrogen (NOx) of vehicles with different regression coefficient $K_{i, j}^{e}$. The regression coefficient $K_{i, j}^{e}$ was calibrated by Ahn et al. [32] using field data collected at Oak Ridge National Laboratory. The calibration regression coefficients have been widely used in a variety of studies $[21,36]$ to assess fuel consumption and transport emissions, as shown in Table 2.

\section{Simulation Analysis}

The application of CAVs in different traffic scenarios, and the improvement of fuel consumption and traffic emissions is different. Therefore, this section designs three different traffic scenarios, a basic segment with bottleneck zone, a ramp of the freeway, and a signalized intersection, to study the impact of CAVs on fuel consumption and emissions.

4.1. Simulation Settings. To explore the impact of CAVs on fuel consumption and traffic emissions of different traffic scenarios, three typical traffic scenarios, including a basic segment with bottleneck zone (Scenario 1 ), a ramp of the freeway (Scenario 2), and a signalized intersection (Scenario 3), are selected in this study. A simulation environment is developed to verify the impact of different penetration rates of CAVs on the fuel consumption and traffic emissions of the transportation system. The penetration rate of CAVs 


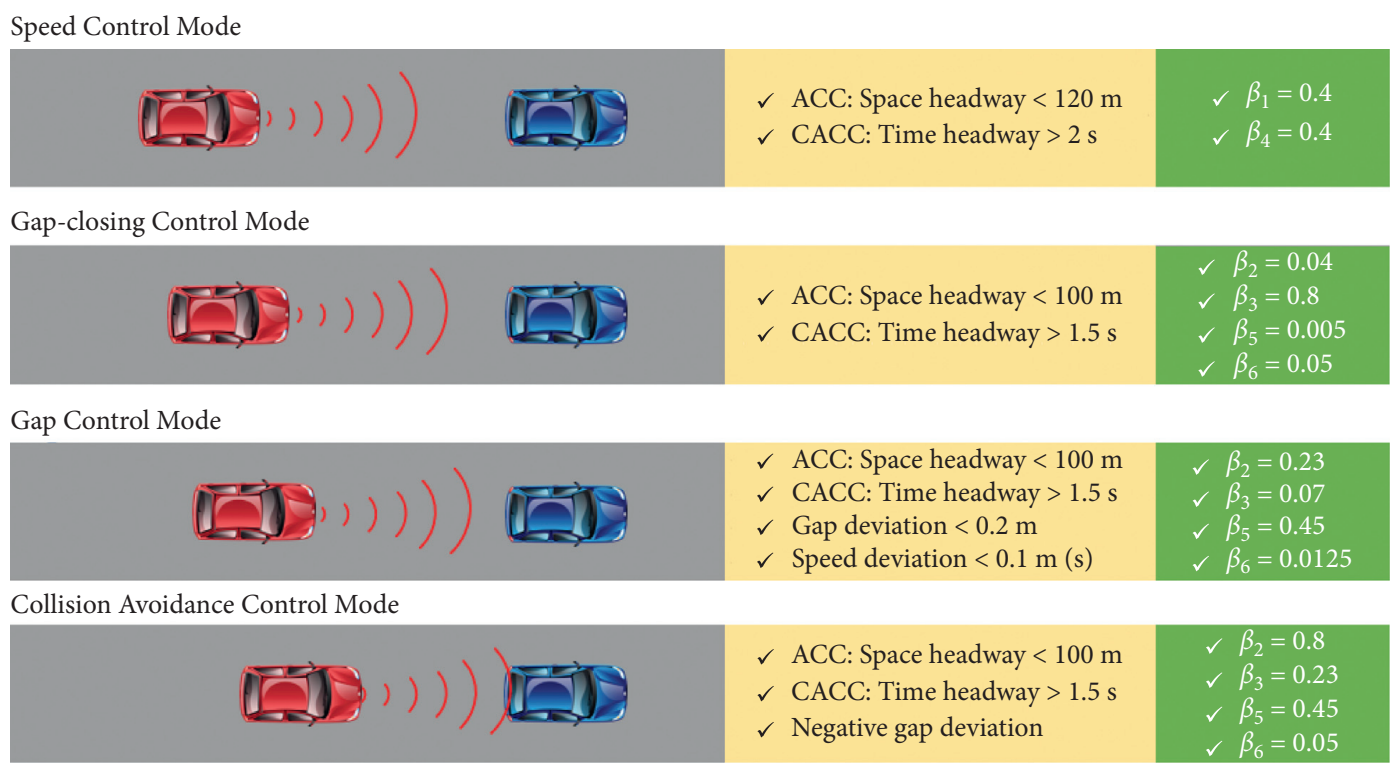

FIGURE 2: Modes of CACC car-following algorithm.

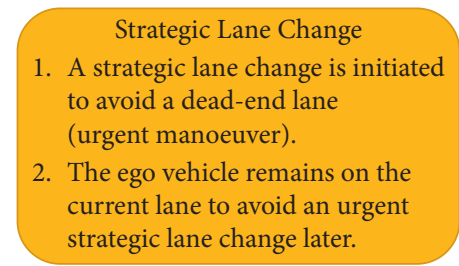

Strategic Lane Change

A strategic lane change is initiated to avoid a dead-end lane current lane to avoid an urgent
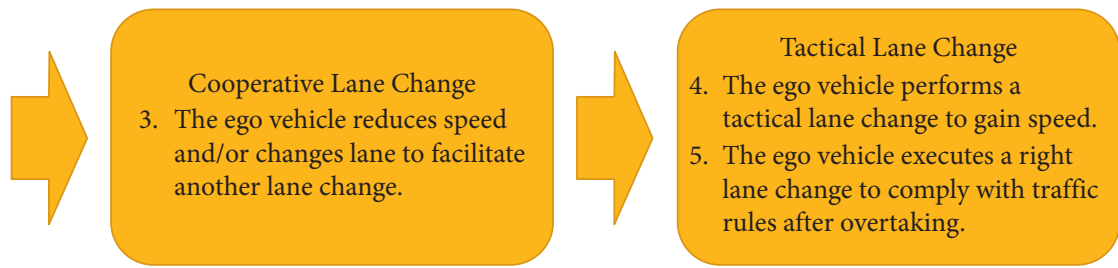

Figure 3: Hierarchy of lane change logic.

TABLE 2: The regression coefficient $K_{i, j}^{e}$ in equation (9).

\begin{tabular}{lcccc}
\hline Parameters & Fuel & CO & HC & NOx \\
\hline$K_{0,0}^{e}$ & -0.679439 & 0.887447 & -0.728042 & -1.067682 \\
$K_{0,1}^{e}$ & 0.135273 & 0.148841 & 0.012211 & 0.254363 \\
$K_{0,2}^{e}$ & 0.015946 & 0.03055 & 0.023371 & 0.008866 \\
$K_{0,3}^{e}$ & -0.001189 & -0.001348 & -0.000093243 & -0.000951 \\
$K_{1,0}^{e}$ & 0.029665 & 0.070994 & 0.02495 & 0.046423 \\
$K_{1,1}^{e}$ & 0.004808 & 0.00387 & 0.010145 & 0.015482 \\
$K_{1,2}^{e}$ & -0.000020535 & 0.000093228 & -0.000103 & 0.000131 \\
$K_{1,3}^{e}$ & $5.5409285 \mathrm{E}-8$ & -0.000000706 & 0.000000618 & -000000328 \\
$K_{2,0}^{e}$ & -0.000276 & -0.000786 & -0.000205 & 0.002876 \\
$K_{2,1}^{e}$ & 0.000083329 & -0.000926 & -0.000549 & -0.00005866 \\
$K_{2,2}^{e}$ & 0.000000937 & 0.000049181 & 0.000037592 & 0.00000024 \\
$K_{2,3}^{e}$ & $-2.479644 \mathrm{E}-8$ & -0.000000314 & 0.000000213 & -000001949 \\
$K_{3,0}^{e}$ & 0.000001487 & 0.000004616 & -0.000113 & 0.000321 \\
$K_{3,1}^{e}$ & -0.000061321 & 0.000046144 & 0.00000331 & $-1.257413 \mathrm{E}-8$ \\
$K_{3,2}^{e}$ & 0.000000304 & -0.00000141 & $-1.739372 \mathrm{E}-8$ & \\
$K_{3,3}^{e}$ & $-4.467234 \mathrm{E}-9$ & $8.1724008 \mathrm{E}-9$ & \\
\hline
\end{tabular}

varies from $0 \%$ to $100 \%$ in steps of $10 \%$. The schematic diagrams of the three traffic scenarios are shown in Figure 4. In the simulation experiment, the CAVs environment is constructed by SUMO and Python. First, three car-following models with appropriate parameters are built in SUMO, to describe the car-following behavior of different vehicles. Then, the trajectory data of all vehicles are obtained based on Python via the TRACI interface of SUMO. Finally, fuel consumption and traffic emissions of each scene are calculated based on equation (9). In addition, the simulation duration of all scenarios is $1200 \mathrm{~s}$, and the time step of the simulation experiment is $1 \mathrm{~s}$. To avoid the influence of randomness, the different random seeds are adopted in the same experiment to simulate ten times, and the average value of the ten times simulation experiments is taken as the final result. 


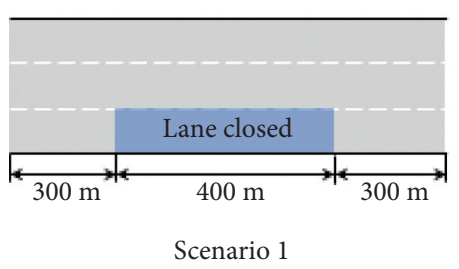

Scenario 1

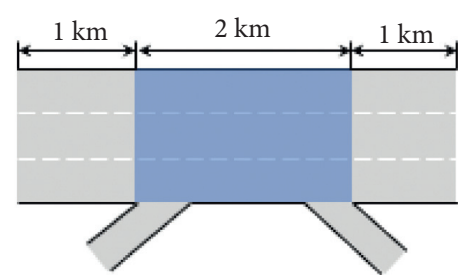

Scenario 2

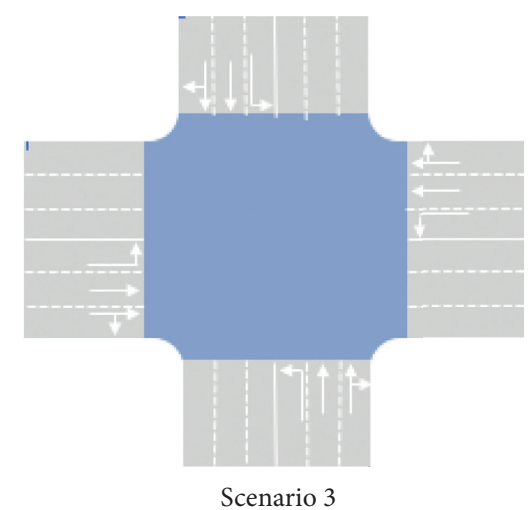

Figure 4: The schematic diagrams of the three traffic scenarios.

\subsection{Results and Discussions}

4.2.1. Basic Road Segment with Bottleneck Zone. The expressway bottleneck zone is usually the sudden point of a traffic accident, and it is a common scenario on the expressway. The road segment is a one-way three-lane expressway of $1 \mathrm{~km}$. Due to accidents or other factors, the rightmost lane is closed at $300 \mathrm{~m}$ to $700 \mathrm{~m}$. This indicates that only two lanes are operating normally. In this scenario, the traffic flow rate of the expressway is $700 \mathrm{veh} / \mathrm{h} / \mathrm{lane}$. Based on the simulation experiments, the fuel consumption and traffic emissions under different penetration rates of CAVs are obtained, as shown in Table 3.

Table 3 reports that when the traffic flow is mixed with CAVs and HDVs, the CO emission is the highest, while the $\mathrm{HC}$ and NOx are lower. Particularly, when the penetration rate of CAVs is $0 \%$, the fuel consumption, $\mathrm{CO}, \mathrm{HC}$, and $\mathrm{NOx}$ are $15.31 \mathrm{~L}, 158.33 \mathrm{~g}, 11.12 \mathrm{~g}$, and $11.56 \mathrm{~g}$, respectively. When the penetration rate of CAVs is $100 \%$, the fuel consumption and traffic emissions are $13.21 \mathrm{~L}, 153.98 \mathrm{~g}, 9.12 \mathrm{~g}$, and $9.32 \mathrm{~g}$, respectively. Moreover, to reflect the variation trend of fuel consumption and traffic emissions with the penetration rate of CAVs, Figure 5 is drawn. Figure 5 shows that fuel consumption and traffic emissions all gradually reduce with the increase of the penetration rate of CAVs. When the penetration rate of CAVs is less than $30 \%$, the decrease of $\mathrm{CO}$ is relatively small, while the decrease of fuel consumption, HC, and NOx are significant, and the trends are consistent. When the penetration rate is low, most of the CAVs are in the adaptive cruise mode, and the normal operation of HDVs needs to be prioritized, so the decline in fuel consumption, $\mathrm{CO}$, and NOx is small. From the simulation results, when the penetration rate is $60 \%-70 \%$, the interference between the HDVs and the CAVs is the smallest, resulting in the maximum decrease in fuel consumption, $\mathrm{CO}$, and $\mathrm{NOx}$ at this stage. With continuous increase, the optimization space for the coordinated control of CAVs is limited, and the decline in fuel consumption, $\mathrm{CO}$, and NOx tends to be flat.

Based on Table 3, the decrease percentage of fuel consumption and traffic emissions with different penetration rates of CAVs, as shown in Table 4 and Figure 6. Figure 6 shows that when the penetration rate of CAVs is less than $30 \%$, the CO emission declines more slowly. Moreover, when the penetration rate of CAVs is greater than $30 \%$, the $\mathrm{CO}$ emission begins to decrease gradually. Compared with $\mathrm{CO}$ emission, the downward trends of fuel consumption, $\mathrm{HC}$, and NOx are more consistent. The analysis shows that there is a bottleneck zone in this scenario. When the traffic flow approaches and leaves the bottleneck zone, the vehicle will cause additional acceleration and deceleration due to lane change, whether HDVs or CAVs. In this scenario, when the penetration rate of CAVs is $100 \%$, the four evaluation indices of fuel consumption, $\mathrm{CO}, \mathrm{HC}$, and NOx decreased by $13.71 \%, 2.75 \%, 17.98 \%$, and $19.36 \%$, respectively. This suggests that the application of CAVs in this scenario has a certain effect on reducing fuel consumption and traffic emissions, but the decline range is small, all less than $20 \%$.

4.2.2. Ramp of Freeway. The ramp of the freeway has a more significant impact on the traffic flow of the main road. The scenario in this section is a basic segment of a one-way threelane of $4 \mathrm{~km}$. At $1 \mathrm{~km}$, there is a ramp where vehicles enter the freeway, and at $3 \mathrm{~km}$, there is a ramp where vehicles leave the freeway. The traffic flow rate of the freeway and ramp are $1600 \mathrm{veh} / \mathrm{h} / \mathrm{lane}$ and $120 \mathrm{veh} / \mathrm{h} / \mathrm{lane}$, respectively. Based on the simulation experiments, the fuel consumption and traffic emissions under different permeability of CAVs are obtained, as shown in Table 5.

Table 5 indicates that CAVs can effectively reduce fuel consumption and traffic emissions (CO, HC, NOx, etc.). When the penetration rate of CAVs is $0 \%$, the fuel consumption and traffic emissions are $35.34 \mathrm{~L}, 348.71 \mathrm{~g}, 25.75 \mathrm{~g}$, and $27.33 \mathrm{~g}$, respectively; when the penetration rate reaches $100 \%$, the fuel consumption and traffic emissions are $32.80 \mathrm{~L}, 341.36 \mathrm{~g}, 22.93 \mathrm{~g}$, and $24.14 \mathrm{~g}$, respectively. This means that compared with traditional HDVs, the widespread use of CAVs can reduce fuel consumption and traffic emissions significantly. Furthermore, the fuel consumption and traffic emissions changes under different penetration rates of CAVs and can be plotted, as shown in Figure 7. Figure 7 reports that when the penetration rate of CAVs is below $90 \%$, fuel consumption and traffic emissions decrease as the penetration rate increases gradually; when the penetration rate of CAVs is greater than $90 \%$, the downward trend in fuel consumption and traffic emissions is more 
TABLE 3: Fuel consumption and traffic emissions under different CAVs penetration rates (Scenario 1).

\begin{tabular}{|c|c|c|c|c|}
\hline Penetration rate $(\%)$ & $\mathrm{FC}(\mathrm{L})$ & $\mathrm{CO}(\mathrm{g})$ & $\mathrm{HC}(\mathrm{g})$ & NOx $(g)$ \\
\hline 0 & 15.31 & 158.33 & 11.12 & 11.56 \\
\hline 10 & 15.13 & 158.29 & 10.94 & 11.37 \\
\hline 20 & 14.88 & 158.27 & 10.65 & 11.05 \\
\hline 30 & 14.56 & 158.12 & 10.31 & 10.67 \\
\hline 40 & 14.33 & 157.42 & 10.10 & 10.45 \\
\hline 50 & 14.11 & 157.42 & 9.87 & 10.17 \\
\hline 60 & 13.95 & 156.63 & 9.73 & 9.98 \\
\hline 70 & 13.61 & 156.04 & 9.40 & 9.63 \\
\hline 80 & 13.55 & 155.32 & 9.38 & 9.62 \\
\hline 90 & 13.35 & 154.68 & 9.21 & 9.41 \\
\hline 100 & 13.21 & 153.98 & 9.12 & 9.32 \\
\hline
\end{tabular}

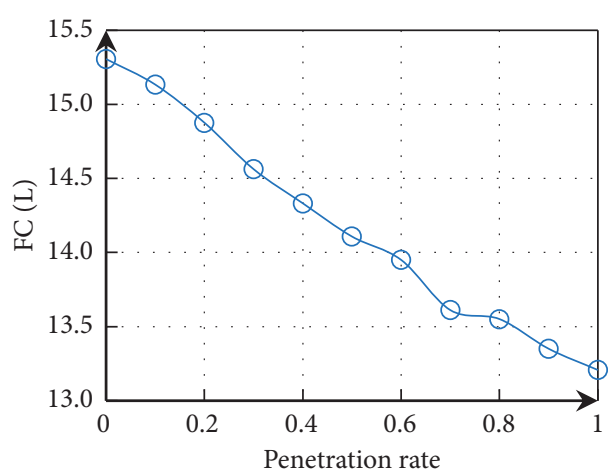

(a)

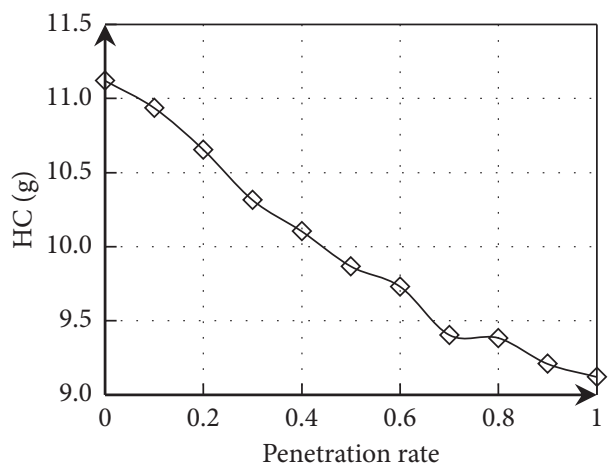

(c)

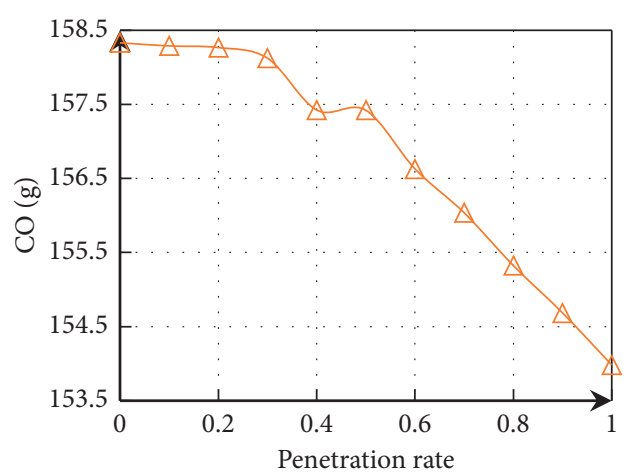

(b)

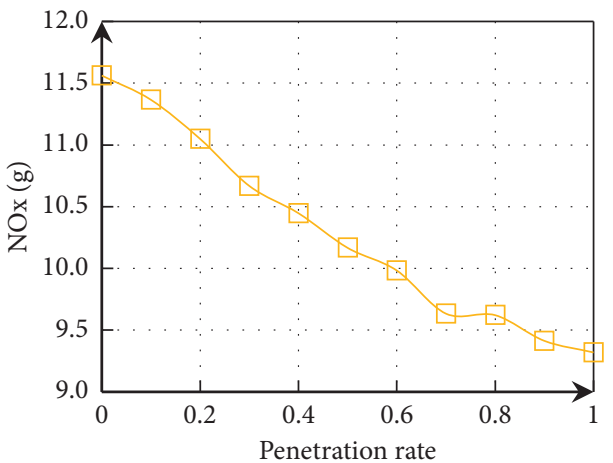

(d)

Figure 5: Fuel consumption and traffic emissions under different penetration rates of CAVs (Scenario 1). (a) Fuel consumption. (b) CO. (c) HC. (d) NOx.

significant. This points out that purely CAVs will have a significant impact on vehicle energy saving and emission reduction in this scenario.

Meanwhile, the decrease in fuel consumption and traffic emissions is obtained based on Table 5, as shown in Table 6 and Figure 8. Figure 8 shows that the effects of CAVs on fuel consumption and traffic emissions are the same. As the penetration rate increases, the overall evaluation indicators show a downward trend. In this scenario, the penetration rate of CAVs has a lower impact on $\mathrm{CO}$ than the other three evaluation indices, and the trend of change is relatively gentle. The reduction percentage of fuel consumption, $\mathrm{CO}, \mathrm{HC}$, and $\mathrm{NOx}$ reaches the maximum when the penetration rate of CAVs is $100 \%$, which are $7.18 \%, 2.11 \%, 10.93 \%$, and $11.68 \%$, respectively.
The result shows that CAVs can maintain a more stable driving speed and minor headway in the road segments. Meanwhile, CACC vehicles form a platoon during operation. Compared with traditional HDVs, the acceleration and deceleration processes are significantly reduced, thus reducing the fuel consumption and emission of traffic flow. However, in this scenario, when vehicles run near the ramp, an HDV or a CAV will be disturbed by the vehicle in the ramp, resulting in different degrees of acceleration and deceleration behavior. Therefore, in the ramp of the freeway, when the penetration rate of CAVs is $100 \%$, compared with $0 \%$, the decline in fuel consumption, $\mathrm{CO}, \mathrm{HC}$, and $\mathrm{NOx}$ are relatively low, all lower than $12 \%$. 
TABle 4: Percentage reduction in fuel consumption and traffic emissions (Scenario 1).

\begin{tabular}{|c|c|c|c|c|}
\hline Penetration rate (\%) & $\mathrm{FC}(\%)$ & $\mathrm{CO}(\%)$ & $\mathrm{HC}(\%)$ & NOx (\%) \\
\hline 0 & NA & NA & NA & NA \\
\hline 10 & 1.13 & 0.03 & 1.65 & 1.70 \\
\hline 20 & 2.81 & 0.04 & 4.19 & 4.44 \\
\hline 30 & 4.86 & 0.13 & 7.24 & 7.73 \\
\hline 40 & 6.36 & 0.57 & 9.14 & 9.64 \\
\hline 50 & 7.83 & 0.58 & 11.27 & 12.07 \\
\hline 60 & 8.86 & 1.08 & 12.50 & 13.66 \\
\hline 70 & 11.07 & 1.45 & 15.43 & 16.68 \\
\hline 80 & 11.47 & 1.90 & 15.62 & 16.78 \\
\hline 90 & 12.78 & 2.30 & 17.19 & 18.58 \\
\hline 100 & 13.71 & 2.75 & 17.98 & 19.39 \\
\hline
\end{tabular}

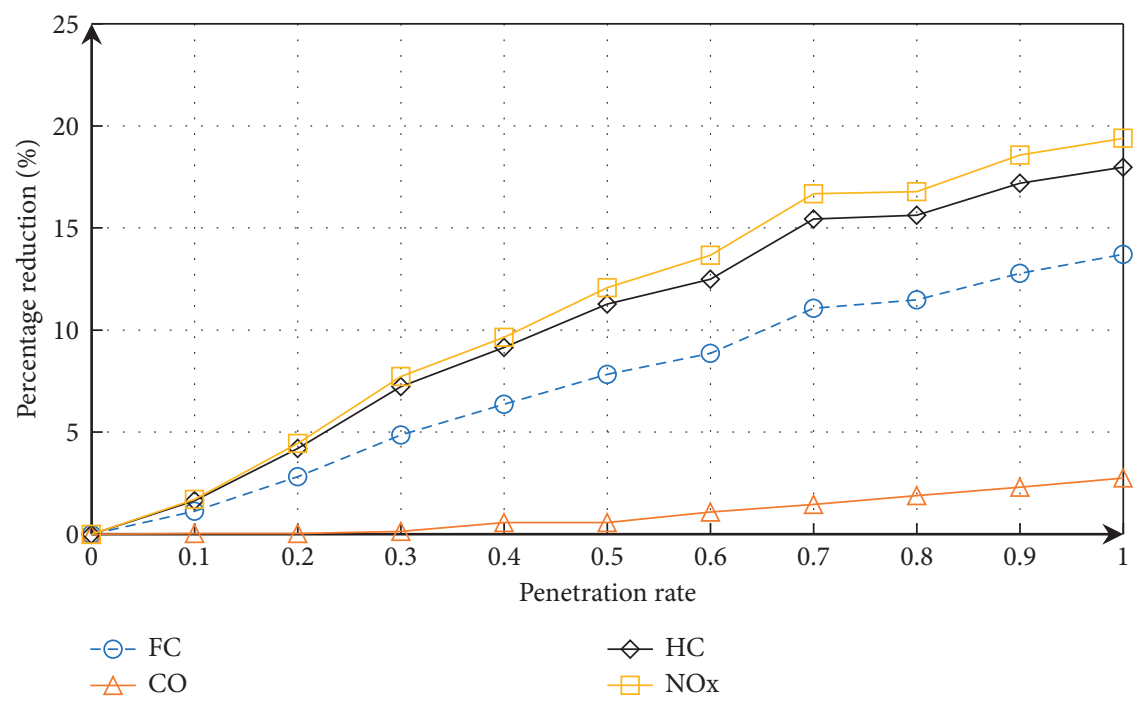

Figure 6: Percentage reduction in fuel consumption and traffic emissions (Scenario 1).

Table 5: Fuel consumption and traffic emissions under different penetration rates of CAVs (Scenario 2).

\begin{tabular}{lcccc}
\hline Penetration rate $(\%)$ & FC $(\mathrm{L})$ & CO $(\mathrm{g})$ & HC $(\mathrm{g})$ & 25.75 \\
\hline 0 & 35.34 & 348.71 & 25.60 & 27.33 \\
10 & 35.20 & 348.13 & 25.38 & 27.10 \\
20 & 35.04 & 348.09 & 25.29 & 26.89 \\
30 & 34.96 & 347.55 & 24.99 & 26.75 \\
40 & 34.73 & 346.97 & 24.69 & 26.48 \\
50 & 34.52 & 346.44 & 24.68 & 26.16 \\
60 & 34.50 & 346.19 & 24.60 & 26.15 \\
70 & 34.49 & 345.49 & 24.35 & 26.05 \\
80 & 34.28 & 345.48 & 24.14 & 25.74 \\
90 & 34.17 & 345.43 & 22.93 & 25.47 \\
100 & 32.80 & 341.36 & 24.14 \\
\hline
\end{tabular}

4.2.3. Signalized Intersection. In urban transportation, the signalized intersection is an essential node for vehicle collection, turning, and evacuation. The setting of signalized intersection scenario is of great significance to the study of the characteristics of traffic flow. The scenario here is a twoway three-lane intersection, including three turns: left turn, straight, and right turn. The traffic flow rate of each lane is $220 \mathrm{veh} / \mathrm{h}$. Based on the simulation experiments, the fuel consumption and traffic emissions under different CAVs conditions in this scenario are obtained, as shown in Table 7.
Table 7 shows that when the penetration rate of CAVs is $0 \%$, the fuel consumption, $\mathrm{CO}, \mathrm{HC}$, and $\mathrm{NOx}$ are $29.74 \mathrm{~L}$, $167.38 \mathrm{~g}, 27.35 \mathrm{~g}$, and $22.47 \mathrm{~g}$, respectively; when the permeability is $100 \%$, the fuel consumption and traffic emissions are $17.58 \mathrm{~L}, 112.36 \mathrm{~g}, 17.49 \mathrm{~g}$, and $14.24 \mathrm{~g}$, respectively. In scenario 3 , as the penetration rate of CAVs increases, fuel consumption and traffic emissions are all showing a downward trend. The changes in fuel consumption and traffic emissions under different penetration rates are shown in Figure 9. Compared with fuel consumption, $\mathrm{HC}$, and $\mathrm{NOx}$, 


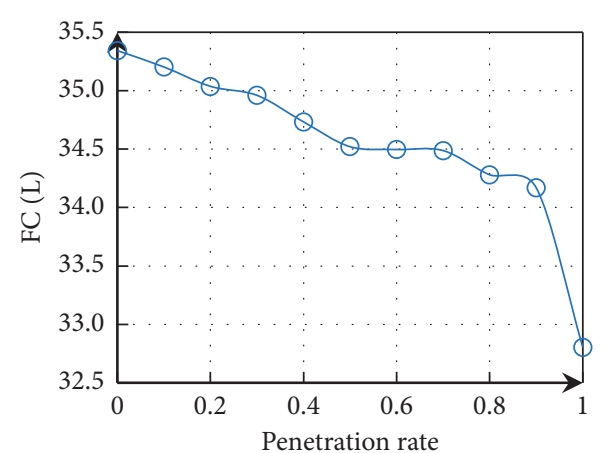

(a)

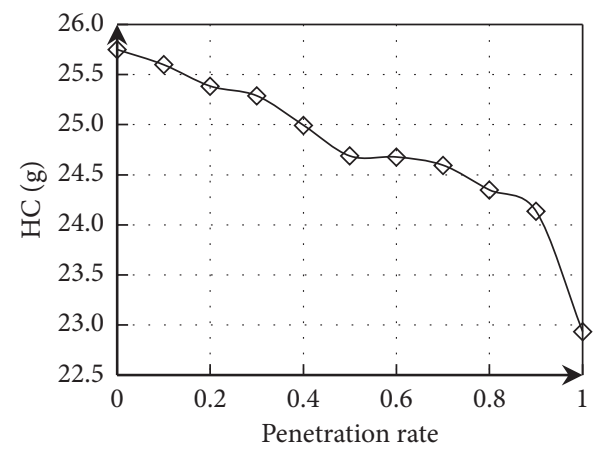

(c)

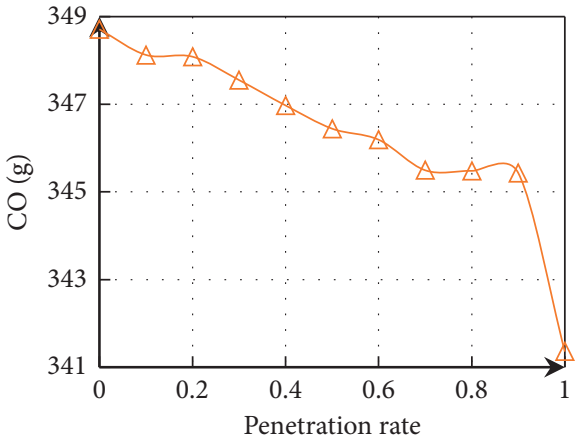

(b)

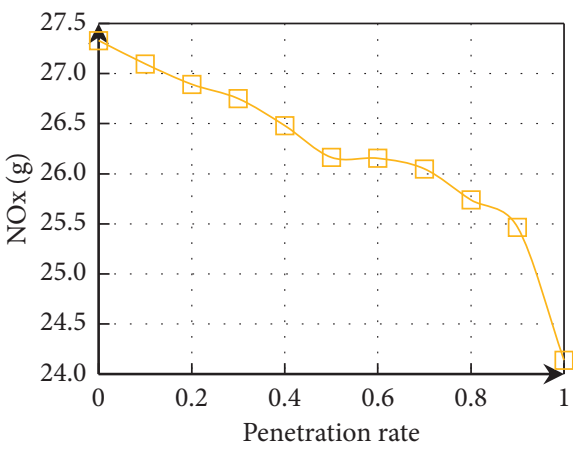

(d)

Figure 7: Fuel consumption and traffic emissions under different penetration rates of CAVs (Scenario 2). (a) Fuel consumption. (b) CO. (c) HC. (d) NOx.

TABle 6: Percentage reduction in fuel consumption and traffic emissions (Scenario 2).

\begin{tabular}{|c|c|c|c|c|}
\hline Penetration rate $(\%)$ & FC (\%) & CO (\%) & $\mathrm{HC}(\%)$ & NOx (\%) \\
\hline 0 & NA & NA & NA & NA \\
\hline 10 & 0.40 & 0.17 & 0.59 & 0.85 \\
\hline 20 & 0.87 & 0.18 & 1.42 & 1.60 \\
\hline 30 & 1.08 & 0.33 & 1.79 & 2.12 \\
\hline 40 & 1.73 & 0.50 & 2.93 & 3.11 \\
\hline 50 & 2.33 & 0.65 & 4.13 & 4.27 \\
\hline 60 & 2.39 & 0.72 & 4.17 & 4.30 \\
\hline 70 & 2.42 & 0.92 & 4.48 & 4.69 \\
\hline 80 & 3.01 & 0.93 & 5.44 & 5.82 \\
\hline 90 & 3.32 & 0.94 & 6.26 & 6.82 \\
\hline 100 & 7.18 & 2.11 & 10.93 & 11.68 \\
\hline
\end{tabular}

CO changes more significantly, and the impact of CAVs on its emission is noticeable. In addition, compared with scenarios 1 and 2, the fuel consumption and traffic emissions in scenario 3 show a linear decrease as the penetration rate of CAVs increases. This means that the use of CAVs can significantly improve fuel consumption and traffic emissions in scenario 3.

Furthermore, the reduction percentages of fuel consumption and traffic emissions under different penetration rates of CAVs is obtained, as shown in Table 8. Meanwhile, Figure 10 provides a more intuitive understanding of the impact of the penetration rate of CAVs on fuel consumption and traffic emissions. It can be seen from Figure 10 that as the penetration rate of CAVs increases, various fuel consumption and traffic emission indicators show a downward trend, and the decline is relatively significant. In scenario 3, the reduction of $\mathrm{CO}$ among the four indicators is slightly lower. When the penetration rate of CAVs is $100 \%$, the percentage of $\mathrm{CO}$ reduction will reach $32.87 \%$, while the declining percentage of fuel consumption, $\mathrm{HC}$, and $\mathrm{NOx}$ reach $40.89 \%, 36.06 \%$, and $36.64 \%$, respectively.

In scenario 3 , the road is relatively more standardized, and there are traffic lights to restrict the traffic flow. Therefore, in this scenario, the advantages of CAVs are more prominent. The four evaluation indicators have a significant decline, reaching more than $32 \%$ when the penetration rate of CAVs is $100 \%$. 


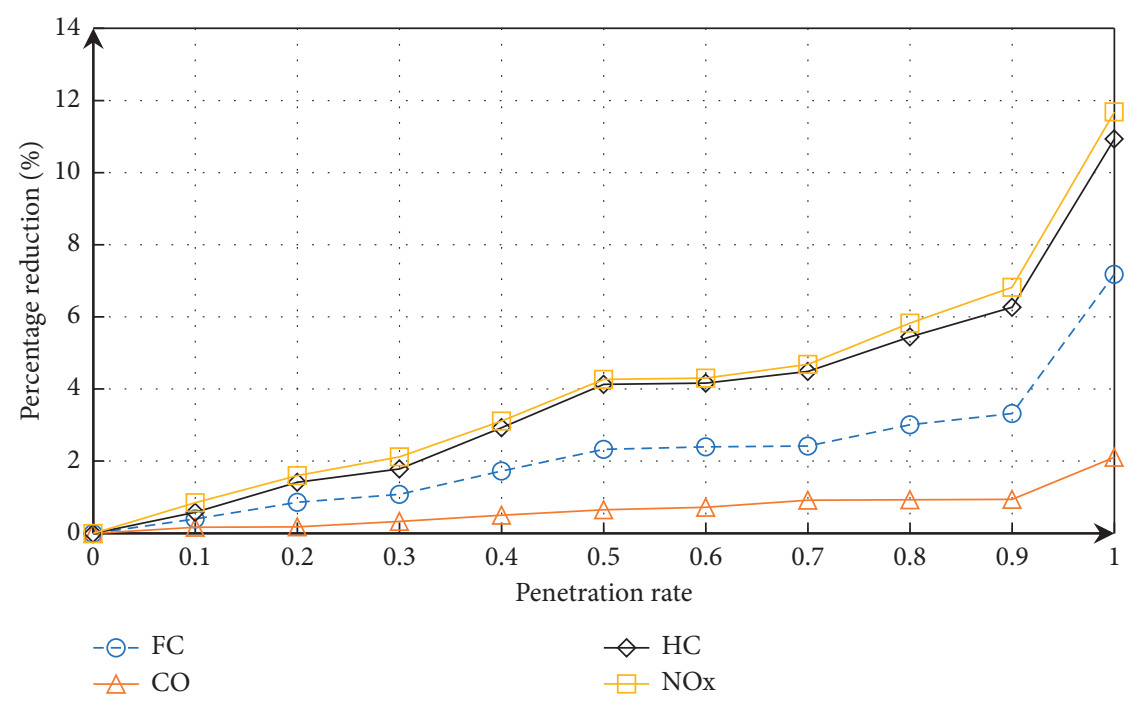

Figure 8: Percentage reduction in fuel consumption and traffic emissions (Scenario 2).

TABle 7: Fuel consumption and emissions under different penetration rates of CAVs (Scenario 3).

\begin{tabular}{lcccc}
\hline Permeability (\%) & FC $(\mathrm{L})$ & CO $(\mathrm{g})$ & HC $(\mathrm{g})$ & \\
\hline 0 & 29.74 & 167.38 & 27.35 & 26.21 \\
10 & 28.55 & 161.80 & 25.05 & 2.47 \\
20 & 27.32 & 156.24 & 24.15 & 20.84 \\
30 & 26.35 & 152.22 & 23.15 & 20.19 \\
40 & 25.27 & 147.76 & 22.01 & 19.48 \\
50 & 24.00 & 142.40 & 20.84 & 18.64 \\
60 & 22.66 & 137.03 & 19.90 & 17.74 \\
70 & 21.51 & 132.04 & 18.91 & 16.98 \\
80 & 20.12 & 125.67 & 18.21 & 16.03 \\
90 & 18.96 & 120.11 & 17.49 & 15.22 \\
100 & 17.58 & 112.36 & & 14.24 \\
\hline
\end{tabular}

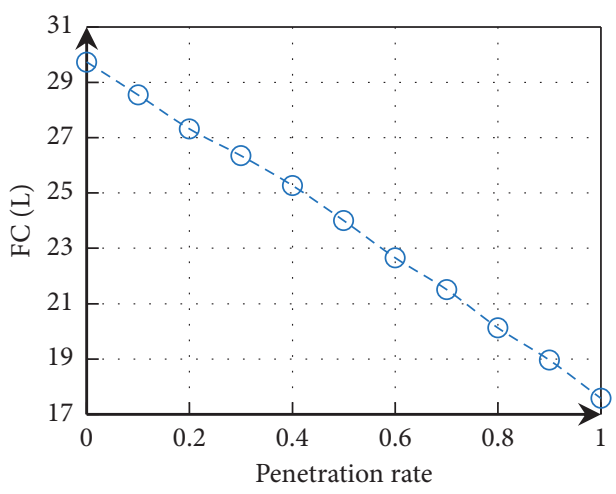

(a)

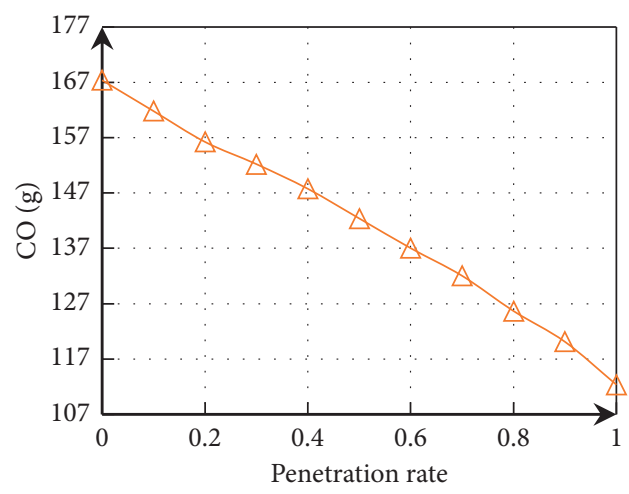

(b)

FIGURE 9: Continued. 


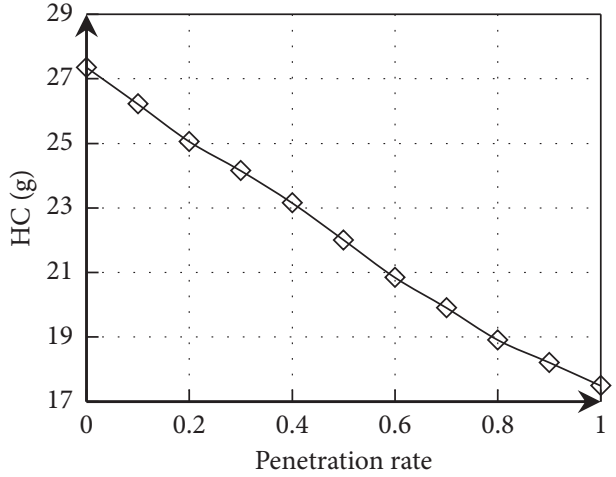

(c)

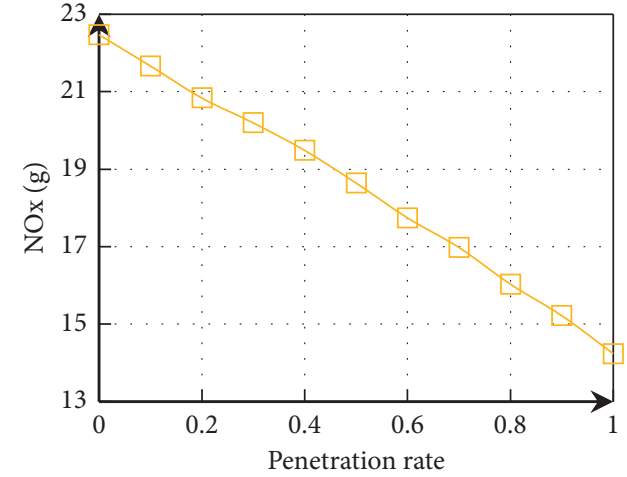

(d)

Figure 9: Fuel consumption and traffic emission for different permeability of CAVs (Scenario 3). (a) Fuel consumption. (b) CO. (c) HC. (d) NOx.

TABle 8: Percentage reduction in fuel consumption and traffic emissions (Scenario 3).

\begin{tabular}{lcccc}
\hline Penetration rate (\%) & FC (\%) & CO (\%) & HC (\%) \\
\hline 0 & NA & NA & NA & NOx (\%) \\
10 & 4.01 & 3.33 & 4.16 & 8.41 \\
20 & 8.14 & 6.65 & 11.71 & 7.59 \\
30 & 11.39 & 9.05 & 15.36 & 10.13 \\
40 & 15.01 & 11.72 & 19.55 & 13.29 \\
50 & 19.29 & 14.92 & 23.80 & 17.04 \\
60 & 23.80 & 18.13 & 27.23 & 21.04 \\
70 & 27.66 & 21.11 & 30.88 & 24.44 \\
80 & 32.34 & 24.92 & -33.43 & -32.63 \\
90 & -36.25 & -28.24 & -36.06 & -36.64 \\
\hline
\end{tabular}

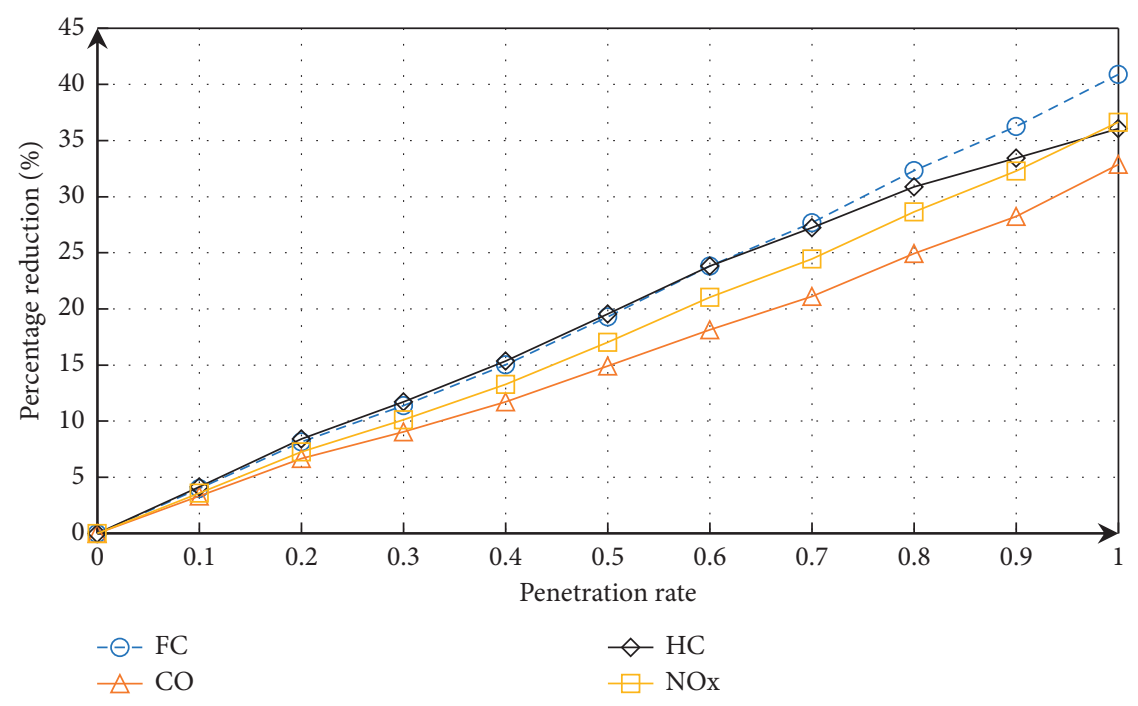

Figure 10: Percentage reduction in fuel consumption and traffic emissions (Scenario 3). 


\section{Conclusion and Policy Implication}

This paper analyzes the fuel consumption and traffic emissions of mixed traffic flow with CAVs in different traffic scenarios. According to the results of the simulation experiment, the conclusions can be obtained as follows:

(1) In different traffic scenarios, CAVs play an effective role in reducing fuel consumption and emissions. With the increase of the penetration rate of CAVs, the declining proportion of fuel consumption and traffic emission increases. This suggests that the widespread application of CAVs can reduce fuel consumption and traffic emission, save resources, and alleviate environmental pollution to a certain extent.

(2) There are certain differences in the impact of CAVs on fuel consumption and traffic emission in different traffic scenarios. In the signalized intersection, the percentage of fuel consumption and traffic emissions reduction both reached more than $32 \%$. However, in the ramp and bottleneck zone of the freeway, when the penetration rate of CAVs is $100 \%$, both fuel consumption and traffic emissions decreased by less than $20 \%$.

(3) Compared with the ramp and bottleneck zone of the freeway, the reduction in fuel consumption and traffic emissions of the signalized intersection is more significant, up to $32 \%$ or more. This means that the application of CAVs has more significant energysaving and emission-reduction effects on urban transportation.

This paper mainly analyzes the mixed traffic flow's fuel consumption and emissions with CAVs and HDVs based on the micro-driving behavior. Thus, the optimization and coordinated control of the CAVs are not considered in this study. Therefore, the collaborative control and optimization of connected automated vehicles can be considered further to reduce fuel consumption and traffic emissions in future work $[37,38]$. Moreover, the trajectory of the CAVs can also be optimized in real-time to achieve the effect of alleviating traffic congestion, fuel consumption, and traffic emissions.

\section{Data Availability}

The data used to support the findings of this study are included in the article.

\section{Conflicts of Interest}

The authors declare that they have no conflicts of interest.

\section{Acknowledgments}

The paper received research funding support from the National Natural Science Foundation of China (52002339), the Sichuan Science and Technology Program (2021YJ0535, 2020YFH0026), the Fundamental Research Funds for the Central Universities (2682021CX058), the Guangxi Science and Technology Program (2021AA01007AA), and the Innovation Center Project of Chengdu Jiao Da Big Data Technology Co., Ltd. (JDSKCXZX202003).

\section{References}

[1] L. Yang, K. W. Chau, W. Y. Szeto, X. Cui, and X. Wang, "Accessibility to transit, by transit, and property prices: spatially varying relationships," Transportation Research Part D: Transport and Environment, vol. 85, Article ID 102387, 2020.

[2] L. Yang, X. Chu, Z. Gou, H. Yang, Y. Lu, and W. Huang, "Accessibility and proximity effects of bus rapid transit on housing prices: heterogeneity across price quantiles and space," Journal of Transport Geography, vol. 88, Article ID 102850, 2020.

[3] Y. Jiang, B. Zhao, M. Liu, and Z. Yao, "A two-level model for traffic signal timing and trajectories planning of multiple CAVs in a random environment," Journal of Advanced Transportation, vol. 2021, p. 13, Article ID 9945398, 2021.

[4] Energy Information Administration, "Use of gasoline - energy explained, your guide to understanding energy - energy information administration," 2018, https://www.eia.gov/ energyexplained/.

[5] Z. Yao, T. Xu, Y. Jiang, and R. Hu, "Linear stability analysis of heterogeneous traffic flow considering degradations of connected automated vehicles and reaction time," Physica A: Statistical Mechanics and Its Applications, vol. 561, Article ID 125218, 2021.

[6] Z. Yao, H. Jiang, Y. Cheng, Y. Jiang, and B. Ran, "Integrated schedule and trajectory optimization for connected automated vehicles in a conflict zone," IEEE Transactions on Intelligent Transportation Systems, 2020.

[7] H. S. Mahmassani, "50th anniversary invited article-autonomous vehicles and connected vehicle systems: flow and operations considerations," Transportation Science, vol. 50, no. 4, pp. 1140-1162, 2016.

[8] S. E. Shladover, C. Nowakowski, X. Y. Lu, and R. Ferlis, "Cooperative adaptive cruise control," Transportation Research Record: Journal of the Transportation Research Board, vol. 2489, no. 1, pp. 145-152, 2015.

[9] M. Wang, S. P. Hoogendoorn, W. Daamen, B. van Arem, B. Shyrokau, and R. Happee, "Delay-compensating strategy to enhance string stability of adaptive cruise controlled vehicles," Transportation Business: Transport Dynamics, vol. 6, no. 3, pp. 211-229, 2018.

[10] J. Rios-Torres and A. A. Malikopoulos, "A survey on the coordination of connected and automated vehicles at intersections and merging at highway on-ramps," IEEE Transactions on Intelligent Transportation Systems, vol. 18, 2017.

[11] R. Scarinci and B. Heydecker, "Control concepts for facilitating motorway on-ramp merging using intelligent vehicles," Transport Reviews, vol. 34, no. 6, pp. 775-797, 2014.

[12] Z. Yao, Y. Wang, B. Liu, B. Zhao, and Y. Jiang, "Fuel consumption and transportation emissions evaluation of mixed traffic flow with connected automated vehicles and humandriven vehicles on expressway," Energy, vol. 230, Article ID 120766, 2021.

[13] “California Air Resources Board," WWW Document, 2002.

[14] L. Ntziachristos and Z. Samaras, COPERT III, Computer Programme to Calculate Emissions from Road Transport, European Environment Agency, København, Denmark, 2000. 
[15] A. Simpson, Parametric Modelling of Energy Consumption in Road Vehicles, University of Queensland, St Lucia, Australia, 2005.

[16] S. Cernuschi, M. Giugliano, A. Cemin, and I. Giovannini, "Modal analysis of vehicle emission factors," The Science of the Total Environment, vol. 169, 1995.

[17] K. Ahn, Microscopic Fuel Consumption and Emission Modeling (Thesis), Virginia Polytechnic Institute and State University, Blacksburg, VA, USA, 1998.

[18] S. Chandra and F. Camal, "A simulation-based evaluation of connected vehicle technology for emissions and fuel consumption," Procedia Engineering, vol. 145, pp. 296-303, 2016.

[19] X. Han, R. Ma, and H. M. Zhang, "Energy-aware trajectory optimization of CAV platoons through a signalized intersection," Transportation Research Part C: Emerging Technologies, vol. 118, Article ID 102652, 2020.

[20] H. Yao and X. Li, "Decentralized control of connected automated vehicle trajectories in mixed traffic at an isolated signalized intersection," Transportation Research Part C: Emerging Technologies, vol. 121, Article ID 102846, 2020.

[21] Y. Qin, H. Wang, and B. Ran, "Stability analysis of connected and automated vehicles to reduce fuel consumption and emissions," Journal of Transportation Engineering, Part A: Systems, vol. 144, no. 11, Article ID 4018068, 2018.

[22] Y. Qin, H. Wang, and B. Ran, "Impacts of cooperative adaptive cruise control platoons on emissions under traffic oscillation," Journal of Intelligent Transportation Systems, vol. 25, no. 4, pp. 376-383, 2021.

[23] E. Mintsis, D. Koutras, K. Porfyri, E. Mitsakis, and S. Boerma, Modelling, Simulation and Assessment of Vehicle Automations and Automated Vehicles' Driver Behaviour in Mixed Traffic, TransAID Deliverable, 2018.

[24] Z. Yao, R. Hu, Y. Wang, Y. Jiang, B. Ran, and Y. Chen, "Stability analysis and the fundamental diagram for mixed connected automated and human-driven vehicles," Physica A: Statistical Mechanics and Its Applications, vol. 533, Article ID 121931, 2019.

[25] Z. Yao, R. Hu, Y. Jiang, and T. Xu, "Stability and safety evaluation of mixed traffic flow with connected automated vehicles on expressways," Journal of Safety Research, vol. 75, pp. 262-274, 2020.

[26] M. Treiber, A. Hennecke, and D. Helbing, "Congested traffic states in empirical observations and microscopic simulations,” Physical Review, vol. 62, no. 2, pp. 1805-1824, 2000.

[27] V. Milanés and S. E. Shladover, "Modeling cooperative and autonomous adaptive cruise control dynamic responses using experimental data," Transportation Research Part C: Emerging Technologies, vol. 48, pp. 285-300, 2014.

[28] L. Xiao, M. Wang, and B. van Arem, "Realistic car-following models for microscopic simulation of adaptive and cooperative adaptive cruise control vehicles," Transportation Research Record: Journal of the Transportation Research Board, vol. 2623, no. 1, pp. 1-9, 2017.

[29] V. Milanés, S. E. Shladover, J. Spring, C. Nowakowski, H. Kawazoe, and M. Nakamura, "Cooperative adaptive cruise control in real traffic situations," IEEE Transactions on Intelligent Transportation Systems, vol. 15, no. 1, pp. 296-305, 2014.

[30] L. Xiao, M. Wang, W. Schakel, and B. van Arem, "Unravelling effects of Cooperative Adaptive Cruise Control deactivation on traffic flow characteristics at merging bottlenecks," Transportation Research Part C: Emerging Technologies, vol. 96, pp. 380-397, 2018.
[31] H. Liu, X. Kan, S. E. Shladover, X.-Y. Lu, and R. E. Ferlis, "Impact of cooperative adaptive cruise control on multilane freeway merge capacity," Journal of Intelligent Transportation Systems, vol. 22, no. 3, pp. 263-275, 2018.

[32] K. Ahn, H. Rakha, A. Trani, and M. Van Aerde, "Estimating vehicle fuel consumption and emissions based on instantaneous speed and acceleration levels," Journal of Transportation Engineering, vol. 128, pp. 182-190, 2002.

[33] R. Akcelik, "Efficiency and drag in the power-based model of fuel consumption," Transportation Research Part B: Methodological, vol. 23, no. 5, pp. 376-385, 1989.

[34] J. N. Hooker, "Optimal driving for single-vehicle fuel economy," Transportation Research Part Policy Pract, vol. 22, pp. 183-201, 1988.

[35] H. Rakha and K. Ahn, "Closure to "estimating vehicle fuel consumption and emissions based on instantaneous speed and acceleration levels" by kyoung Ahn, hesham rakha, antonio trani, and michel van aerde," Journal of Transportation Engineering, vol. 129, no. 5, pp. 579-581, 2003.

[36] T. Q. Tang, H.-J. Huang, and H. Y. Shang, "Influences of the driver's bounded rationality on micro driving behavior, fuel consumption and emissions," Transportation Research Part D: Transport and Environment, vol. 41, pp. 423-432, 2015.

[37] Z. Yao, B. Zhao, T. Yuan, H. Jiang, and Y. Jiang, "Reducing gasoline consumption in mixed connected automated vehicles environment: a joint optimization framework for traffic signals and vehicle trajectory," Journal of Cleaner Production, vol. 265, Article ID 121836, 2020.

[38] S. K. Zegeye, B. De Schutter, J. Hellendoorn, E. A. Breunesse, and A. Hegyi, "Integrated macroscopic traffic flow, emission, and fuel consumption model for control purposes," Transportation Research Part C: Emerging Technologies, vol. 31, pp. 158-171, 2013. 